\title{
All-Inkjet-Printed Vertical Heterostructure for Wafer-Scale Electronics
}

Dong Un Lim, ${ }^{1 \dagger}$ Seungbeom Choi ${ }^{2 \dagger}$ Seongchan Kim, ${ }^{2}$ Young Jin Choi, ${ }^{2}$ Sungjoo Lee, ${ }^{2}$ Moon Sung Kang, ${ }^{4}$ Yong-Hoon Kim, ${ }^{2,3, *}$ Jeong Ho Cho ${ }^{1, *}$

${ }^{1}$ Department of Chemical and Biomolecular Engineering, Yonsei University, Seoul 03722, Korea.

${ }^{2}$ SKKU Advanced Institute of Nanotechnology (SAINT), ${ }^{3}$ School of Advanced Materials Science and Engineering, Sungkyunkwan University, Suwon 440-746, Korea.

${ }^{4}$ Department of Chemical and Biomolecular Engineering, Sogang University, Seoul 04107, Korea.

*Corresponding author: J. H. Cho (jhcho94@yonsei.ac.kr) and Y.-H. Kim (yhkim76@,skku.edu)

$\dagger$ D. U. Lim and S. Choi contributed equally to this work. 


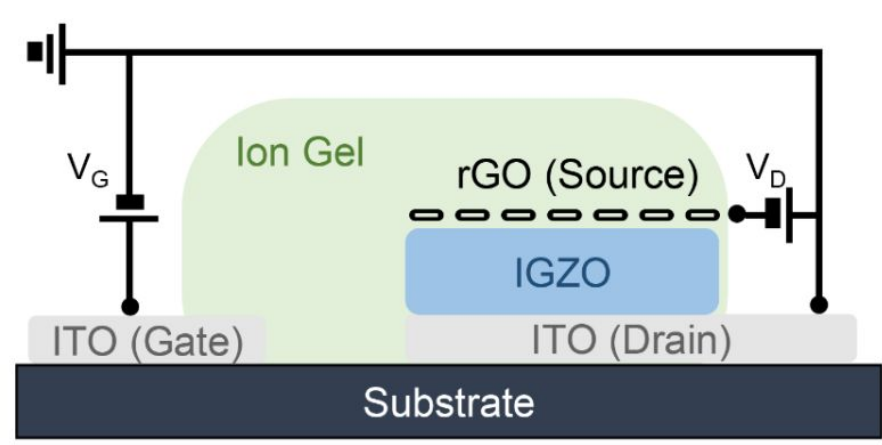

Figure S1. Schematic cross-sectional image of all-inkjet-printed vertical SB transistors.

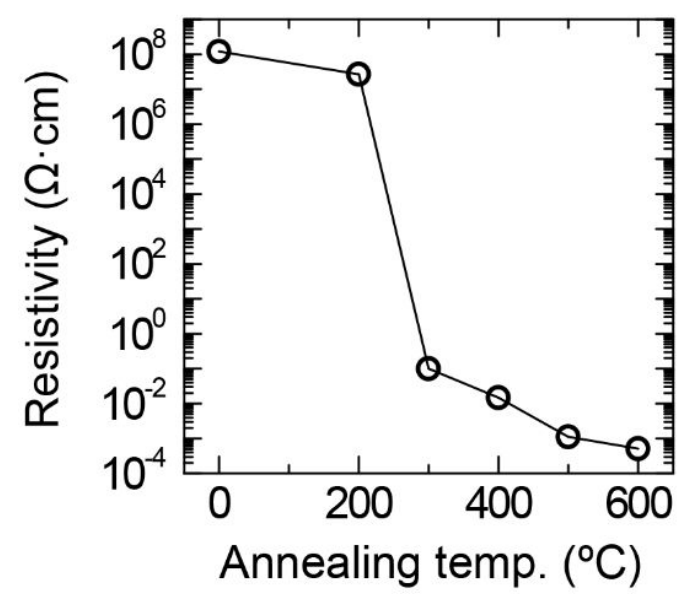

Figure S2. Electrical resistivity of inkjet-printed ITO electrode as a function of annealing temperature.

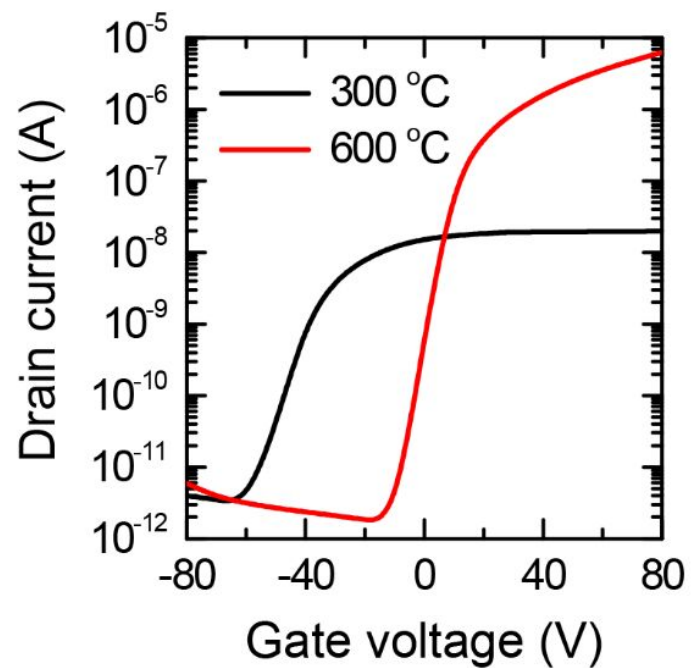

Figure S3. Transfer characteristics of IGZO transistor based on inkjet-printed IGZO channel annealed at 300 and $600{ }^{\circ} \mathrm{C}$ with 300 -nm-thick $\mathrm{SiO}_{2}$ gate dielectric. 


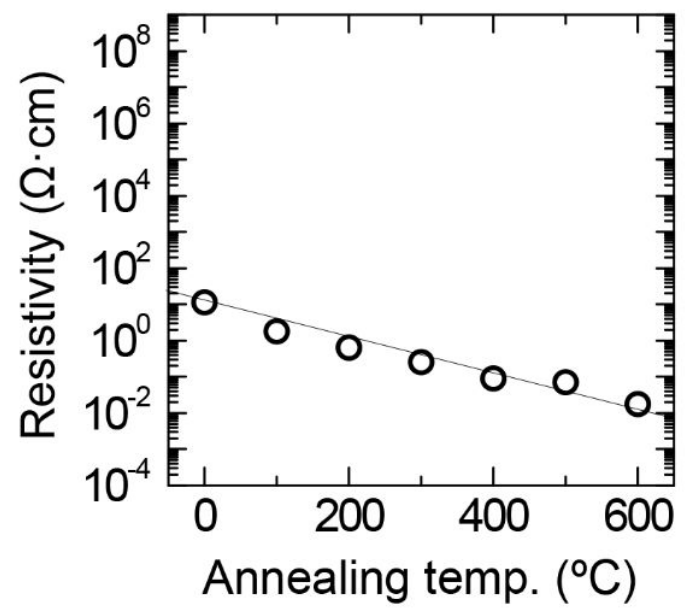

Figure S4. Electrical resistivity of inkjet-printed rGO electrode as a function of annealing temperature.

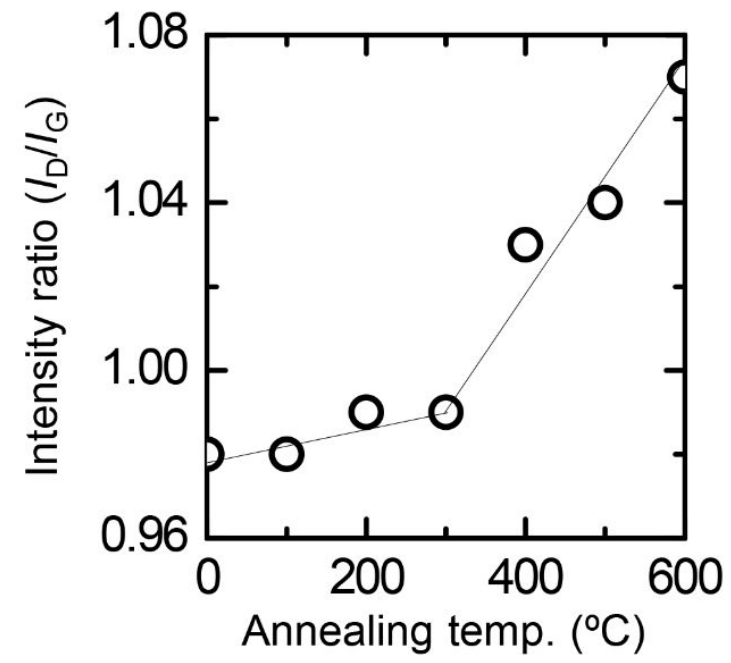

Figure S5. Raman intensity ratio $\left(I_{\mathrm{D}} / I_{\mathrm{G}}\right)$ of inkjet-printed $\mathrm{rGO}$ electrode as a function of annealing temperature. 

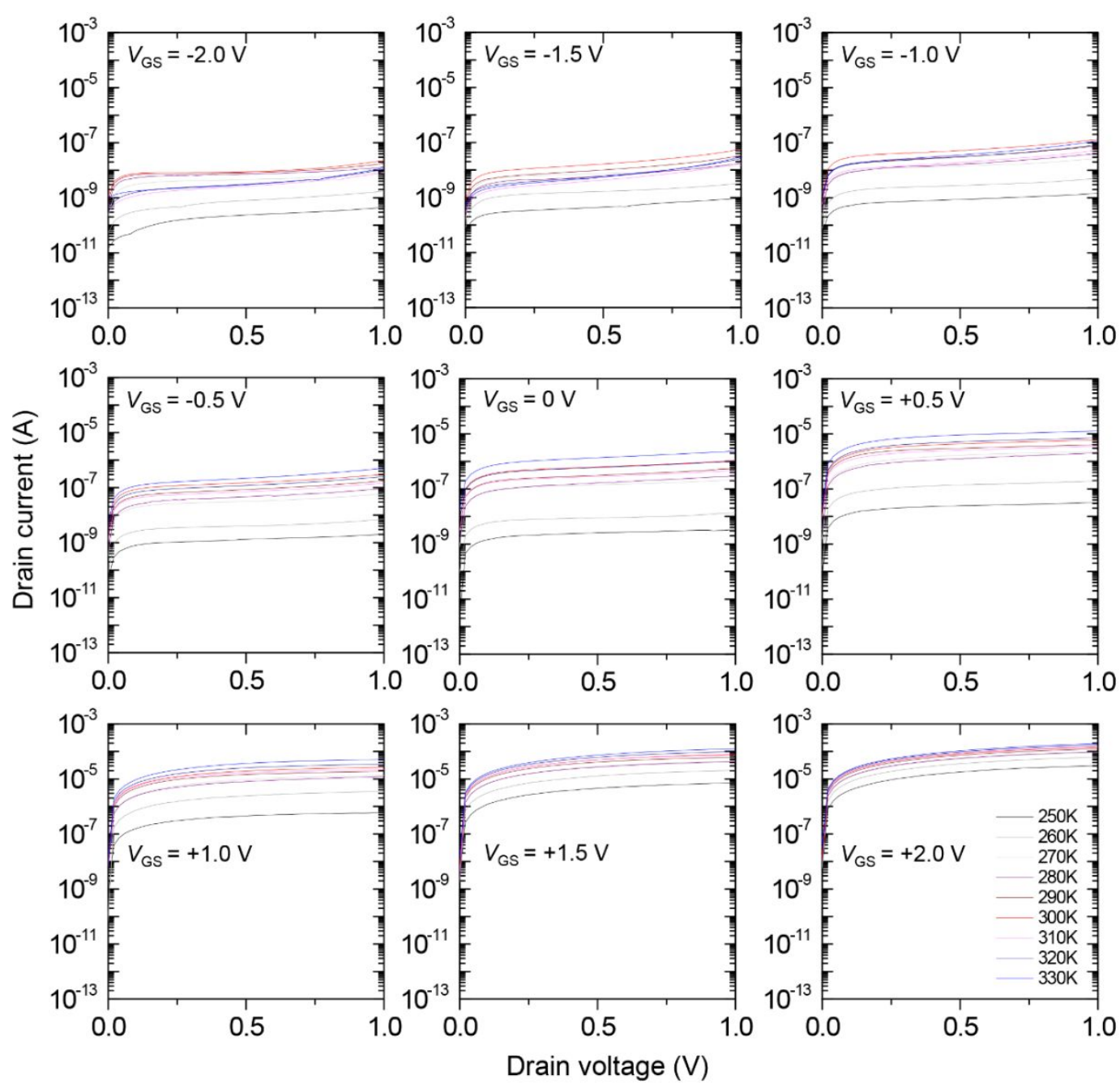

Figure S6. Temperature-dependent output curves of vertical SB transistor measured at different $V_{\mathrm{GS}}$ values.

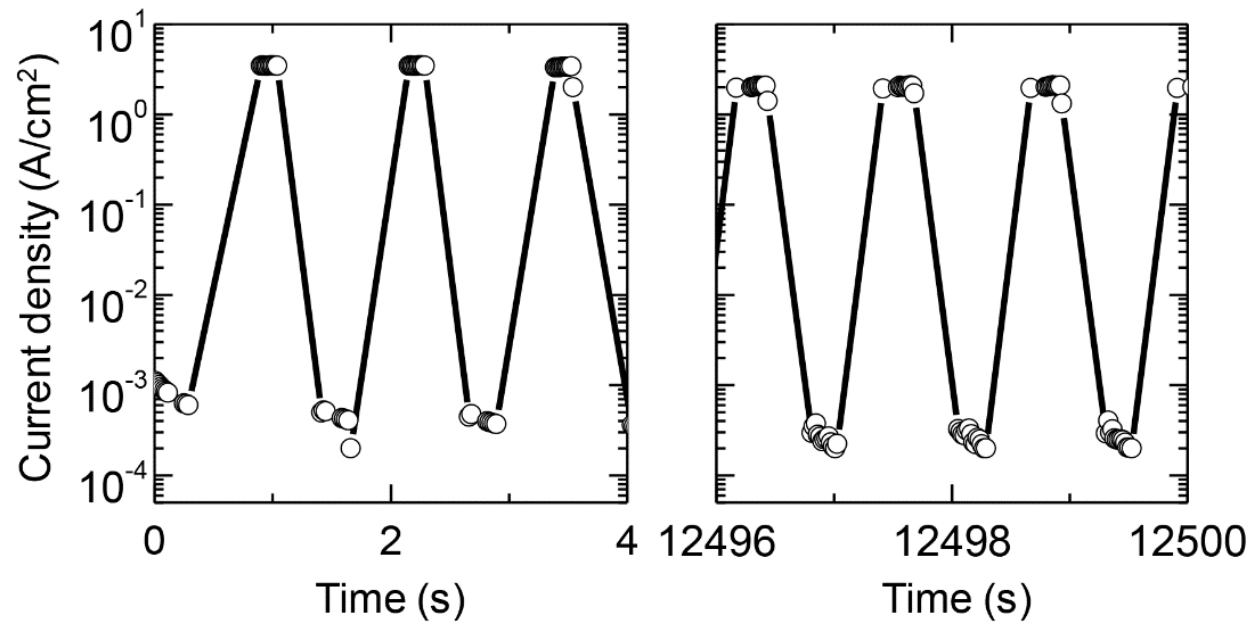

Figure S7. Magnified plot of the initial and the final regions of the cyclic test in Figure $3 \mathrm{~d}$. 

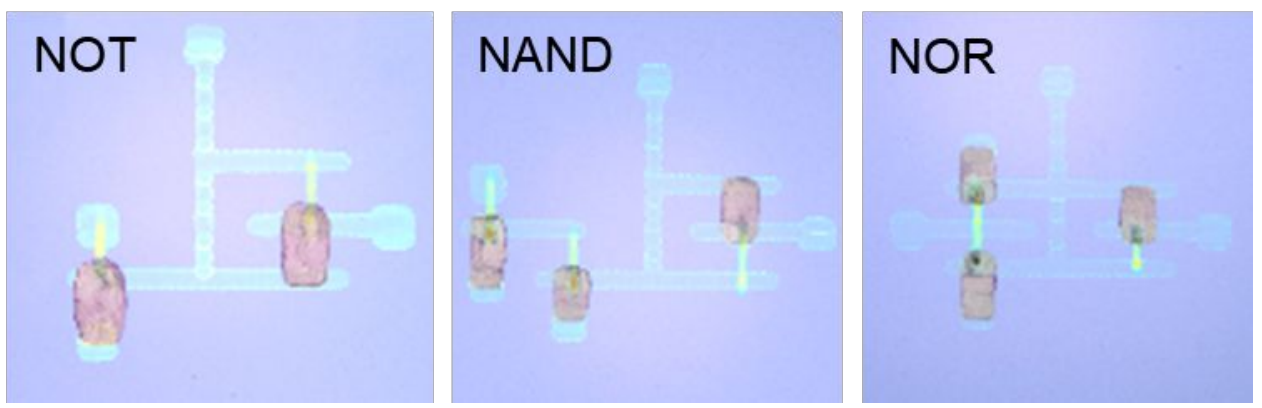

Figure S8. OM images of all-inkjet-printed NOT, NAND, and NOR gates. 